\title{
Neue Erkenntnisse zum Wirken von Tilemann Stella (1525-1589)
}

\author{
Gyula Pápay ${ }^{1}$
}

Received: 30 November 2018 / Accepted: 24 January 2019 / Published online: 12 August 2019

(c) The Author(s) 2019

\section{Zusammenfassung}

Tilemann Stella (1525-1589) gehörte zu den bedeutendsten Kartographen des 16. Jahrhunderts. Er schuf wichtige Werke vor allem auf dem Gebiet der topographischen Kartographie, aber auch bezüglich der Verlagskartographie und der thematischen Kartographie. Seine Rezeption in der kartographischen Literatur wird insbesondere durch die komplizierte Quellenlage beeinträchtigt. Daher ist es erfreulich, dass vor allem dank der Ermittlung bisher unbekannter Quellen und zum Teil durch Neubewertung bereits bekannter Quellen neue Erkenntnisse über seine Tätigkeit gewonnen werden können. Der vorliegende Beitrag beleuchtet die finanziellen Entstehungsumstände Stellas berühmter Deutschlandskarte aus dem Jahre 1560. Stella verfasste mehrere Studien zu der Methodik der großmaßstäbigen Kartierung, aber in der deutschen kartographischen Literatur ist es nicht bekannt, dass er eine Skizze zur Erklärung seiner Kartierungsmethodik anfertigte. Über die Globuskartographie Stellas waren auch noch vor kurzer Zeit äußerst geringe Informationen vorhanden. Das betrifft auch seinen Beitrag zur Weiterentwicklung des kartographischen Zeichensystems.

Schlüsselwörter Kartographiegeschichte $\cdot$ Kartierungsmethodik · Globuskartographie $\cdot$ Kartographische Semiotik

\begin{abstract}
New Knowledges into the Work of Tilemann Stella (1525-1589)

Tilemann Stella (1525-1589) was one of the most important cartographers of the 16th century. He created important works, especially in the field of topographic cartography, but also in terms of publishing cartography and thematic cartography. His reception in the cartographic literature is particularly affected by the complicated source situation. Therefore, it is gratifying that new insights into his work can be gained, thanks in particular to the identification of previously unknown sources and partly to the revaluation of already known sources. The present article explains the financial circumstances of Stella's famous map of Germany from the year 1560. Stella wrote several studies on the methodology of large-scale mapping, but it is not known in the German cartographical literature that he made a sketch to explain his mapping methodology. Very little information was available lately about Stellas' globe cartography. This also applies to his contribution to the further development of the cartographic sign system.
\end{abstract}

Keywords Cartography history $\cdot$ Mapping methodology $\cdot$ Globe cartography $\cdot$ Cartographic semiotics

\section{Einführung}

Der Todestag von Tilemann Stella, der zu den bedeutendsten Kartographen des 16. Jahrhunderts gehört, jährt sich in diesem Jahr bereits zum 430. Mal. Seine umfangreiche kartographische Tätigkeit wurde in der kartographischen Literatur vielfach, aber immer noch nicht hinreichend gewürdigt.

\section{Gyula Pápay}

gyula.papay@uni-rostock.de

1 University of Rostock, Rostock, Germany
Das hängt in erster Linie mit einem enormen Quellenverlust, aber auch damit zusammen, dass die Publikation über sein Wirken in vielen Fällen nicht im kartographischen Rahmen erfolgte, sondern in Zeitschriften mit geringer Verbreitung (vgl. u. a. Cordshagen 1986, 1989, 1990 und Pápay 1989, 1990). Eine Ausnahme bildet in dieser Hinsicht die von Ruthar Oehme und Lothar Zögner 1989 veröffentlichte Publikation (vgl. Oehme/Zögner). Seit dem Erscheinen dieser umfassenden Studie wurden weitere Beiträge veröffentlicht (vgl. u. a. Brichzin 1990, 1993; Meurer 2001; Pápay 2018). Die Intention dieses Artikels besteht nicht darin, die seitdem 
gewonnenen Erkenntnisse zusammenfassend zu wiedergeben, sondern einige Aspekte der Tätigkeit Stellas darzulegen, die bisher nicht bekannt waren oder in der kartographischen Literatur noch nicht hinreichend gewürdigt wurden.

\section{2 „Die gemeine Landtaffel des Deutschen Landes..." (1560)}

Diese in nur wenigen Exemplaren ${ }^{1}$ überlieferte Karte Deutschlands aus dem Jahre 1560 gehört zu den bedeutendsten Deutschlandkarten des 16. Jahrhunderts und zugleich zu den bekanntesten Karten Stellas. Die Karte enthält eine Widmung für den Herzog zu Mecklenburg Johann Albrecht (1525-1576), die auch in dem zu dieser Karte verfassten Begleitbuch „Kurtzer und klarer bericht vom gebrauch und nutz der newen Landtaffeln..." wiederholt wird. Aus diesen Textpassagen geht hervor, dass die Entstehung der Karte von dem Herzog gefördert wurde. Die konkrete Form der herzoglichen Unterstützung blieb bisher jedoch unbekannt. Bei den Recherchen im Landesarchiv Schwerin fand der Verf. im September 2018 in dem Ausgabenverzeichnis des Herzogs Johann Albrecht einen Vermerk vom 7. Februar 1558, woraus hervorgeht, dass diese Karte in Handzeichnung bereits 1558 fertig gestellt war, wovon vermutlich der Herzog eine Kopie erhielt. Der Vermerk lautet wie folgt: „140 thaler dem Tylemanno Stella fur den Globum Mappa vnnd Horologiu(m) auch außgelegt Zerung vnd fur 30 thaler bucher zu kauff(en). Swerin im 7 Februarii““ ${ }^{2}$ Mit der Bezeichnung „Globum Mappa“ ist eine Globularkarte, d. h. eine runde Karte gemeint. Anhand der hier wiedergegebenen Quelle lässt sich die Förderung dieser Karte durch den Herzog konkretisieren. Es handelte sich dabei um eine erhebliche finanzielle Unterstützung, denn das jährliche Durchschnittsgehalt eines Professors betrug damals etwa 150 Taler. Diese Quelle beinhaltet auch noch weitere Informationen über das Vorgehen Stellas bei der Erstellung der Deutschlandkarte. Die Vermerke „Zerung“ und,, bucher“ sind Hinweise darauf, dass Stella zur Überarbeitung der Karte von Sebastian Münster (1488-1552) aus dem Jahre 1525 eine umfangreiche Reisetätigkeit unternehmen und außerdem schriftliche Unterlagen (wahrscheinlich Listen von Ortsentfernungen und astronomische Tabellen) beschaffen musste. Die Deutschlandskarte von Münster und ebenso

\footnotetext{
${ }^{1}$ Lediglich fünf erhaltene Exemplare sind nachweisbar: Sammlung Niewodniczanski (Bitburg); Kirchenbibliothek (Bad Neustadt an der Aisch); Privatbesitz in den USA; Germanisches Nationalmuseum (Nürnberg) und Francisceumsbibliothek (Zerbst).

${ }^{2}$ Landeshauptarchiv Schwerin. 2.22-1 Renterei, Nr.0/61, Register 1555-1569, Gemeine Ausgaben. Bei der korrekten Umschreibung war Ernst Münch (Professor für Geschichte des Mittelalters/Mecklenburgische Landesgeschichte an der Universität Rostock) behilflich.
}

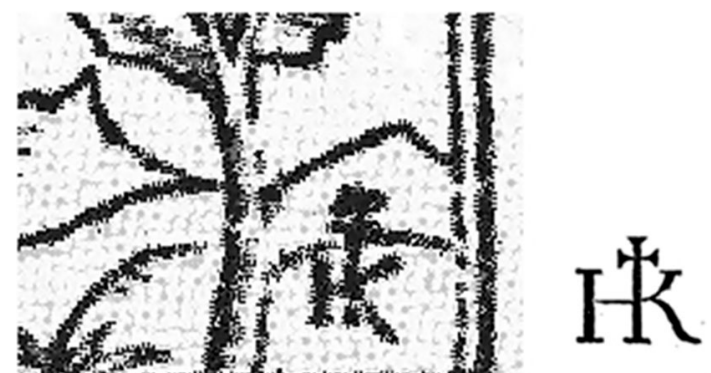

Abb. 1 Druckerzeichen in der Karte „Die gemeine Landtaffel des Deutschen Landes..." und das Druckerzeichen von Hans Klimm

die Karte von Stella sind mit astronomischen Darstellungen kombiniert, die teilweise von Stella aktualisiert wurden. An den Blattecken befinden sich vier astronomische Kreisfiguren. Die oben links angebrachte Figur dient zur Bestimmung des Sonnenauf- und -untergangs. Die zweite oben rechts, in der der Polarstern erscheint, dient zur Zeitbestimmung während der Nacht. Der „Circkel“ unten links enthält von Stella aktualisierte Kalenderbestimmungen für den Zeitraum von 1556 bis 1609. Die in der unteren rechten Ecke befindliche Figur mit astrologischer Funktion trägt die Bezeichnung „Circkel des Horoscopi vnd Planeten stvnd“. Der Kalenderring um die Karte enthält die folgenden Angaben: Buchstaben für die Bezeichnung der Tage, Heiligennamen, Monatsnamen, Tierkreisskala mit den Tierkreiszeichen sowie die Bilder und die Namen des Tierkreises.

Wer der Formschneider der Deutschlandskarte war, galt in der kartographischen Literatur bisher als ungeklärt. In dem „Allgemeines Lexikon der bildenden Künstler...“ (Bd. 37, S. 407) ist dafür der Formschneider Hans Klimm genannt, jedoch von P. H. Meurer wird diese Angabe in Zweifel gezogen (vgl. Meurer, S. 307). Die Abb. 1 zeigt das Druckerzeichen in der Stella-Karte und das Druckerzeichen von Hans Klimm, der um 1560 als Formschneider in Wittenberg wirkte.

\section{Stellas Skizze zur Erklärung seiner Kartierungsmethode (1569)}

Die Deutschlandkarte von 1560 betrachtete Stella als ,geringe“ Vorstufe zu der ,grossen beschreibung des Deutschen landes“, zu deren Anfertigung er „kein fleis und mühe“ zu unterlassen versprach (vgl. Stella 1560, S. 1). Die Konzeption hierfür publizierte Stella in seinem „Methodus...“ 1566. Das hier konzipierte Vorhaben verfolgte Stella bis zu seinem Lebensende, aber vollenden konnte er nicht. Seine umfangreiche Materialsammlung ist verloren gegangen. Stella verfasste neben dem „Methodus..." auch noch andere methodische QuerySchriften, die jedoch nicht publiziert wurden. 


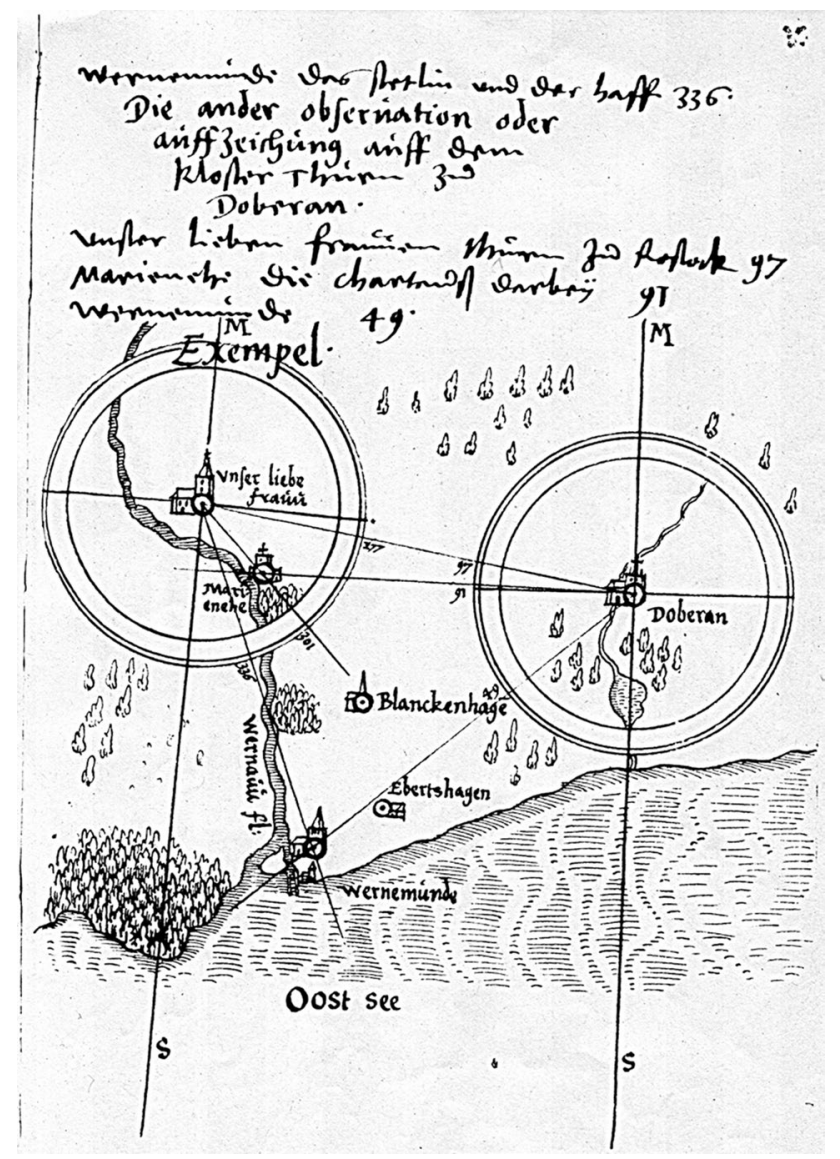

Abb. 2 Stellas Skizze zur Illustration seiner Vermessungsmethode (1569)

Zwei Studien befinden sich im Staatsarchiv Schwerin (vgl. Cordshagen 1989, 1990).

Im Archiv der Universitätsbibliothek von Aberdeen wird eine prächtige Ledermappe mit Widmung an den Mecklenburger Herzog Ulrich III. (1527-1603): „Ulricus Dei Gracia Dux Megalburgensis“ (MS 149) aufbewahrt (vgl. Stewart 1973/74). Diese Mappe beinhaltet Manuskripte, die von Tilemann Stella verfasst wurden, darunter einen „Bericht“, in dem seine Kartierungsmethode beschrieben wird. Die wissenschaftshistorische Einmaligkeit dieses „Berichtes“, den Stella 1569 verfasste, besteht darin, dass hier Stella zu seinen Darlegungen auch eine kartographische Skizze beifügte, um seine Vermessungsmethodik zu illustrieren. Keine seiner anderen Studien enthalten eine solche graphische Erklärung. Die Universität Aberdeen stellte dankenswerterweise eine Kopie von dieser Illustration zur Verfügung (Abb. 2). Das Manuskript Stellas gelangte durch den Studenten John Johnston (vor 1570-1611), der 1584 die Universität Rostock besuchte, nach Schottland. Johnston war ein bedeutender schottischer Dichter und Professor an der University of St Andrews.

\section{Stellas Globuskartographie}

\subsection{Erd- und Himmelsglobus (1553)}

Stella entfaltete eine vielfältige kartographische Tätigkeit, zu der auch die Globuskartographie gehörte. Auf der Grundlage der überlieferten Quellen kann man Stella lediglich die Anfertigung von zwei Globen zuschreiben. Der erste und größere Globus entstand durch einen Auftrag des Herzogs Johann Albrecht in den Jahren 1552 und 1553. Der zweite und wesentlich kleinere Globus (Durchmesser $27.5 \mathrm{~cm}$ ) ist der Weißenburger Himmelsglobus aus dem Jahre 1555, der eine Widmung an den Kurfürsten August von Sachsen enthält. Da der größere Globus verschollen war, galt dieser Globus bis vor kurzem als der einzige überlieferte Globus Stellas und fand eine gebührende Würdigung (Fauser 1973; Hamel 2018; und Pápay 2018).

Eine wichtige Quelle über Stellas Globuskartographie, die selbst von Stella stammt, ist erst vor kurzem bekannt geworden. Es handelt sich um einen Brief, den Stella in Rostock am 20. Januar 1553 in lateinischer Sprache an Melanchthon verfasste. Der Fundort dieses Briefes ist Paris, Bibliothèque Sainte Geneviève (Paris BSG, Ms. 1458, f. 67 $\left.67^{\mathrm{v}}-69^{\mathrm{r}}\right){ }^{3}$ Die Melanchthon-Forschungsstelle der Heidelberger Akademie stellte den Inhalt des Briefes online zur Verfügung. ${ }^{4}$ Stella bezeichnete in seinem Brief den Globus als „caelestem illi ac terrestrem globum". Von der Melanchthon-Forschungsstelle wurde diese Passage so übersetzt, dass Stella „einen Himmels- und einen Erdglobus" für den Herzog erarbeitete. Aus diesem Grund wurde eine frühere Annahme (vgl. Pápay 2016), dass es sich hier um einen Himmels- und Erdglobus in einem handelt, zunächst revidiert (vgl. Pápay 2018, S. 12). Die erneute Überprüfung der betreffenden Passage führte dazu, dass aus dieser Formulierung keine Zweizahl abgeleitet werden kann. Stella erhielt für die Herstellung eines kombinierten Himmels- und Erdglobus vom Herzog „25 marckstuck”. Es handelte sich hier um Markstücke, deren Wert nach der Bemerkung von Stella doppelt so hoch war wie der eines Joachimstalers. ${ }^{6}$ Da der Joachimstaler seit

\footnotetext{
3 "Sed primas debeo illustrissimo principi Ioanni Alberto, sub cuius celsitudine quam laudabilis clementia et veneranda sit humilitas, dici non potest. Pro munere dedit 25 marckstuck (Sic vocant genus numismatis) quaelibet bis est Ioachimici, et quia adornaturus est bibliothecam, egit mecum, ut caelestem illi ac terrestrem globum, eosque iusta magnitudine conficiam. Studio et clementia principis motus hunc laborem libenter suscepi, et fere ad mediam partem perduxi." Für diese Information gilt ein besonderer Dank der Leiterin der Melanchthon-Forschungsstelle, Christine Mundhenk.

${ }^{4}$ MBW..., Regestnummer: 6715. https://www.haw.uni-heidelberg .de/forschung/forschungsstellen/melanchthon/mbw-online.de.html.

5 Durch Christiane Reitz, Professorin für Lateinische Philologie/Latinistik an der Universität Rostock.

6 Übersetzung von Christine Mundhenk.
} 
1536 einen Silbergehalt von $26,39 \mathrm{~g}$ besaß, erhielt Stella vom Herzog eine beträchtliche Summe, die wohl für die Herstellung eines großen Globus ausreichend war. Der Brief an Melanchthon macht es weiterhin deutlich, welchen großen Einfluss Melanchthon auf die kartographische Tätigkeit Stellas ausübte. Dass Stella, den Globus eigenhändig gemalt hat, lässt sich quellenmäßig durch eine Ode belegen, die der Melanchthonschüler Petrus Vicentius (1510-1581) anlässlich der Hochzeit Stellas (Vicentius and Petrus 1554) verfasste (vgl. Vicentius, nicht paginiert, siebte Seite).

Stella verfasste über die Globuskartographie auch eine theoretische Schrift mit dem Titel: „Tilemanni Stella explicatio, et canones globi coelestis, terrestrisq[ue], ac viatorij. in fol[io] minori, sine operculo [= ohne Deckel] zusammen geheft, Sub signo (abnehmender Mond) 56. " Der Hinweis auf diese verloren gegangene Studie wurde erst kürzlich aufgefunden, in dem handschriftlichen Katalog der Bibliothek von Johann Albrecht, der im Jahre 1573 von Samuel Fabricius zusammengestellt wurde. Über den Verbleib des großen Erd- und Himmelsglobus sind in Mecklenburg keine Quellen vorhanden.

Die letzte Quelle zu den globuskartographischen Aktivitäten Stellas stammt aus dem Jahre 1560. Johann Albrecht, von dem Stella eine lebenslange Anstellung erhielt, beantragte für Stellas sämtliche Publikationen ein Druckprivileg, das von dem Kaiser erteilt wurde (vgl. Kaiserliches Privileg 1560/Meurer 2001, S. 330). 1569 wurde dieses Privileg verlängert und wurde auch hier der Globus erwähnt. $\mathrm{Zu}$ dieser Zeit befasste sich Stella jedoch nicht mehr mit Globuskartographie. Das geht eindeutig aus dem Verlängerungsantrag hervor, den Johann Albrecht am 14. Januar 1569 verfasste (vgl. Voltelini 1894, S. LXIX). Über die Tätigkeit Stellas in der Zeit von 1560 bis 1585 liegen mehrere Quellen im Staatsarchiv Schwerin und in der Sächsischen Landesbibliothek vor. Auf der Grundlage dieser Quellen kann man zweifelsfrei feststellen, dass Stellas globuskartographische Tätigkeit auf die erste Hälfte der 1550er-Jahre begrenzte. Er betrachtete die große Deutschlandkarte als sein Lebenswerk und führte seine Materialsammlung bis zu seinem Tode im Jahre 1589 fort.

Die oben dargelegten Quellen liefern nur wenige Anhaltspunkte zu der Beschaffenheit des Erd- und Himmelsglobus Stellas. Mit ziemlich großer Sicherheit kann man annehmen, dass seine Konzeption für eine Kombination der Erddarstellung mit astronomischen Inhalten auf der Deutschlandkarte Münsters basierte.

Neue Erkenntnisse zur Struktur des verschollen gegoltenen Globus Stellas wurden durch zwei Ereignisse initiiert. Von dem St. Galler Globus, der in dem Schweizerischen Landesmuseum in Zürich aufbewahrt wird, wurde mit großem Aufwand von 2007 bis 2009 eine Replik für St. Gallen angefertigt, wo sich der Globus bis 1712 befand. Dazu entstand eine ausführliche Dokumentation (vgl. Rohrbach,
M./Gnädinger, B., 2009). Im Juni 2015 erwarb die Zentralbibliothek Zürich ein auf Kalbspergament mit Aquarell, Gouache und Goldfarbe koloriertes, gemaltes Abbild des St. Galler Globus in einem Format von $59 \times 44 \mathrm{~cm}$ (Nachfolgend als Globuspergament bezeichnet.) Jost Schmid, der Leiter der Abteilung Karten und Panoramen der Zentralbibliothek Zürich, wurde durch diesen interessanten Fund dazu veranlasst, die Erforschung der Genese des St. Galler Globus zu intensivieren. Er erkannte mehrere Indizien für eine Beziehung zu dem lutherischen Norddeutschland bzw. zu Mecklenburg. Ihm gilt bezüglich der Annahme, dass unter anderen auch Tilemann Stella als Autor in Frage kommen könnte, die Priorität. Quellen- und Literaturhinweise für die Fokussierung auf Tilemann Stella wurden vom Verf. an die Kartensammlung der Schweizerischen Nationalbibliothek geliefert (vgl. Pápay 2016). Jost Schmid veröffentlichte mehrere Publikationen über den St. Galler Globus (vgl. Schmid 2016, 2017), die letzte erschien in der „Cartographica Helvetica“ (vgl. Schmid-Lanter 2018). Diese Publikationen enthalten einerseits eine sehr gute Analyse des St. Galler Globus, aber auch Hypothesen, die Überprüfungen bedürfen. 2016 wurden von dem Globus durch Infrarotreflektographie und Radiographie Aufnahmen angefertigt, wodurch auf dem Gestell übermalte Porträts sichtbar gemacht wurden. Ein nur schemenhaft erkennbares Porträt wurde von Schmid dem Herzog Johann VII. von Mecklenburg (1576-1592) zugeordnet, und davon ausgehend entwarf er eine Entstehungsgeschichte des Globus, wofür keinerlei historischen Quellen vorliegen. ${ }^{7}$

Mit ziemlich großer Sicherheit kann man annehmen, dass es sich bei dem St. Galler Globus um den Globus handelt, den Tilemann Stella 1552/53 in Rostock ${ }^{8}$ erstellte. Nach 1569 erfolgte jedoch eine Aktualisierung der kartographischen Darstellung mit Hilfe der Mercatorkarte von 1569. Die Aktualisierung kann man aus mehreren Gründen nicht mit Stella in Verbindung bringen. Erstens war er nach 1569 globuskartographisch nicht mehr tätig. Zweitens hätte Stella die inkorrekte Darstellung der mecklenburgischen Ostseeküste, von der er sehr gute kartographische Informationen besaß, niemals von der Mercatorkarte übernommen.

\footnotetext{
7 Im Internet sind zahlreiche Eintragungen vorhanden, die auf die Publikationen von Schmid basieren und daher eine Korrektur bedürfen. Beispiele: https://de.wikipedia.org/wiki/St._Galler_Globus .https ://blog.nationalmuseum.ch/2017/09/geheimnis-des-st-galler-globu s-gelueftet.

www.siwiarchiv.de/st-galler-globus-wird-tilemann-Stella-zugeschrieben. https://de.wikipedia.org/wiki/Tilemann_Stella.

${ }^{8}$ Dass Stella den Globus in Rostock erstellte und nicht in Schwerin, belegt ein Brief von Jakob Bordinger vom 22. Juli 1553 aus Rostock an Johann Albrecht: „Tilemannus Stella opus suum sphaericum iamdudum absolvit". Zitiert nach Schirrmacher, Friedrich Wilhelm: Johann Albrecht I..., S. 761. Auf dem Meridianring wird Rostock mit „Rostoch“ geschrieben, so wie Stella und andere Gelehrten in ihren lateinischsprachigen Publikationen Rostock bezeichnet haben.
} 


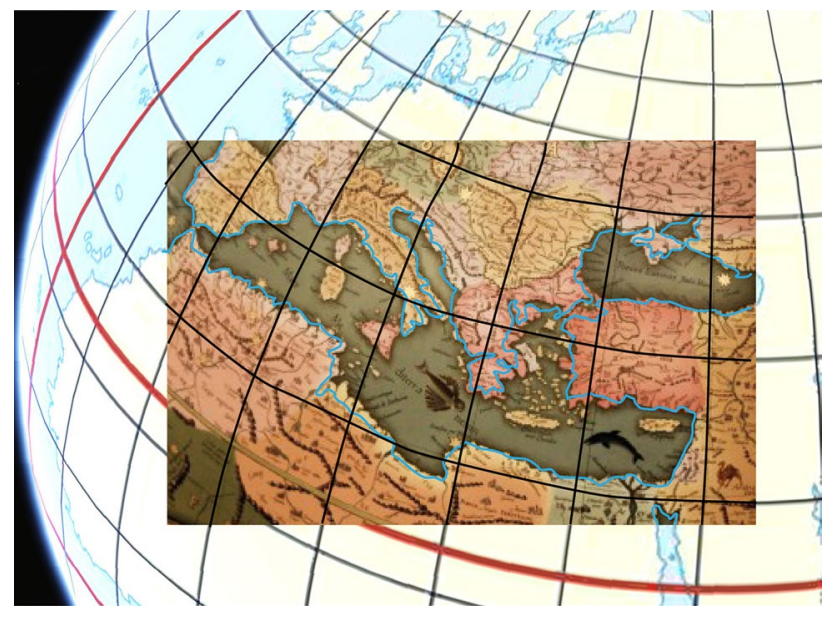

Abb. 3 Von der Mercatorkarte 1569 angefertigter virtueller Globus, in den eine Teilaufnahme des St. Galler Globus eingefügt ist

Drittens: In dem Globus wurde Rostock erst nachträglich ohne Ortsignatur ergänzt, ${ }^{9}$ da Rostock in der Mercatorkarte nicht vorhanden war, aber Rostock auf dem Meridianring genannt wurde. Bei der Durchleuchtung des Globus wurden keine Farbreste von der ursprünglichen Bemalung gefunden. Dieser Umstand widerspricht jedoch nur scheinbar der Übermalungshypothese, denn die Änderungen waren so umfassend, dass eine Neugrundierung erforderlich wurde. Von wem, wann und wo diese Aktualisierung vorgenommen worden war, ist nicht bekannt.

\subsection{Kartographische Analyse des Globuspergaments}

Obwohl das Globuspergament keine Angaben zu seiner Entstehungszeit und zu dem Entstehungszusammenhang enthält, diente es nach Jost Schmid-Lanter einer VerkaufsVorschau und seine Entstehung wurde auf den 1. November 1595 gesetzt (vgl. u. a. Schmid-Lanter 2018, S. 36).

Um die kartographische Struktur des Globuspergaments in Bezug auf den St. Galler Globus analysieren zu können, wurde vom Verf. eine Vektorkarte von den Küstenlinien und dem Gradnetz der Mercatorkarte von 1569 erstellt. Diese Karte wurde dann in eine Rasterdatei umgewandelt und zu ihrer Betrachtung mit Google Earth in eine KML-Datei überführt. ${ }^{10}$ Der Vergleich mit dem St. Galler Globus zeigte, dass die Karte auf den Globus kartographisch sehr korrekt übertragen wurde. Weiterhin ist es aus diesem Vergleich

\footnotetext{
${ }^{9}$ Für diese Information gilt Jost Schmid Dank.

${ }^{10}$ Die kml-Datei wurde von Mátyás Gede (Eötvös University Budapest, Department of Cartography and Geoinformatics/Virtual Globes Museum) erstellt.
}

ersichtlich, dass der virtuelle Globus zur Untersuchung der kartographischen Struktur des St. Galler Globus verwendet werden kann (Abb. 3). Die Gegenüberstellung des Globus von Mercator aus dem Jahre 1541 mit dem ,virtuellen Globus von 1569“ macht den kartographischen Erkenntnisgewinn bezüglich der Ost-West-Ausdehnung von Europa und Afrika in diesem Zeitraum sichtbar (Abb. 4). Diese Abweichung liefert die Grundlage zur Bestimmung des Entstehungszeitraums des Globuspergaments. Die Abb. 5 zeigt das Globuspergament zwischen dem Mercatorglobus 1541 und dem ,virtuellen Globus von 1569“, in dem die kartographische Struktur mit der Struktur des St. Galler Globus übereinstimmt. Diese Gegenüberstellung zeigt deutlich, dass der Globus von 1541 und das Globuspergament noch wesentlich stärker von dem ptolemäischen Erbe geprägt sind als die Mercatorkarte von 1569. Aus dem Strukturvergleich lässt sich ableiten, dass das Globuspergament den Globus Stellas hinsichtlich der kartographischen Struktur in der ursprünglichen Form, also noch vor der Aktualisierung, zeigt. Dafür spricht auch die Wiedergabe derjenigen Beschriftungen, die auf dem Globuspergament in sphärischer Perspektive erscheinen, aber die auf dem St. Galler Globus nicht in dieser Form vorhanden sind. Man muss aber auch feststellen, dass es sich hier um eine künstlerische Wiedergabe handelt, die nicht frei von Fehlern ist, so z. B. wurden der Äquator, der nördliche Wendekreis und die Ekliptik falsch lokalisiert. Als Grundlage für die Ablehnung der hier dargelegten Hypothese könnten jene Schriftpassagen in dem Globuspergament dienen, die vor der Küste der amerikanischen Kontinente angebracht sind. Sie wurden auch für den St. Galler Globus aus der Mercatorkarte 1569 entnommen. In der Tat ist das ein rätselhafter Umstand, den man nur so erklären kann, dass sie in der ursprünglichen Zeichnung nicht vorhanden waren und erst nach der Übermalung ergänzt wurden.

Infolge der strukturellen Untersuchung des Globuspergaments erhebt sich die Frage, ob es ursprünglich zu einem anderen Zweck als Verkauf-Vorschau angefertigt wurde. Seine Entstehung könnte man auch mit einer anderen Hypothese erklären. Seit den 1550er-Jahren befand sich ein attraktiver Globus, der mehrmals an den Reichstagen ausgestellt wurde, im Besitz der Familie Fugger. Möglicherweise handelte es sich bei diem Globus um jenen, der später als St. Galler Globus bezeichnet wurde. ${ }^{11}$ Der bayerischen Herzog Albrecht V. (1528-1579) erwarb diesen Globus, dessen Inhalt veraltet war und aktualisiert werden sollte, ${ }^{12} \mathrm{im}$ Jahre 1572. Es gibt Indizien dafür, dass die Aktualisierung in München erfolgte. Da Hans Fugger (1531-1598) den Globus

\footnotetext{
${ }^{11}$ Die Augsburger Präsenz des St. Galler Globus hat bereits Franz Grenacher angenommen (vgl. Grenacher 1968).

12 Fürstlich und Gräflich Fuggersches Familien- und Stiftungsarchiv, Dillingen. Briefbücher der Fugger 1.2.6. a Nr. 12 Jan.-Mai 1572.
} 
Abb. 4 Der Mercatorglobus 1541 und der virtueller Globus, der auf die Mercatorkarte 1569 basiert

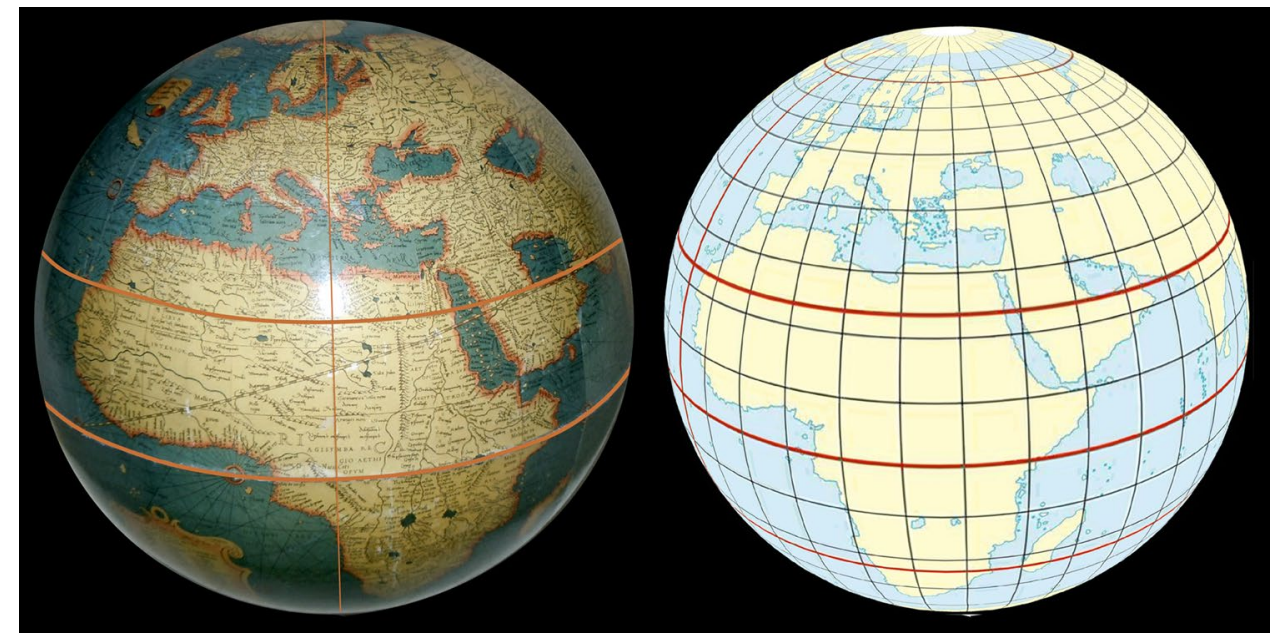

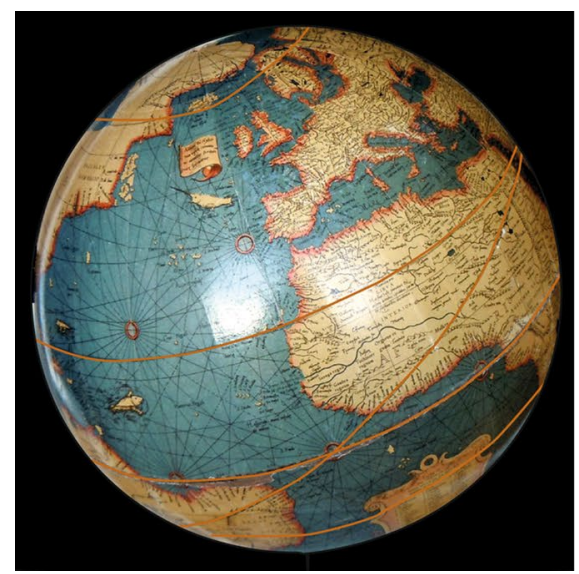
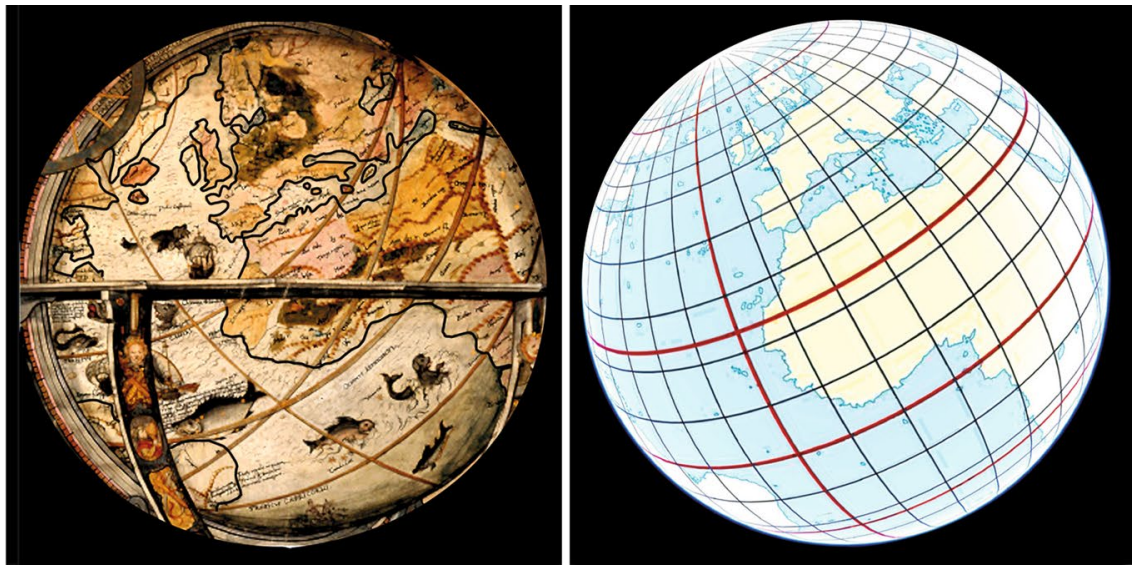

Abb. 5 Der Mercatorglobus 1541, das Globuspergament und der virtuelle Mercatorglobus 1569

sehr ungern dem Herzog überließ, kann man annehmen, dass das Globuspergament möglicherweise als Erinnerungsbild bereits in Nürnberg entstand. Auf Grund dieser Hypothese und der obigen Strukturanalyse ist es wahrscheinlich, dass das Globuspergament Stellas Globus in dem Originalzustand zeigt.

\section{Stellas Beitrag zur Weiterentwicklung des Kartographischen Zeichensystems}

In den Karten des 16. Jahrhunderts wurden die Orte mittels einfacher Ringe oder mit ikonischen Symbolen, d. h. mit kleinen Stadtbildern, dargestellt. Eine Zeichenerklärung war auch dadurch entbehrlich, da eine explizite Ortklassifizierung nicht vorhanden war. Die Verdrängung ikonischer Zeichen durch geometrische Signaturen führte zu einer Modernisierung der kartographischen Darstellung. In diesem Prozess nimmt Tilemann Stella einen wichtigen Platz ein. Seine Karte von Judäa und Israel veröffentlichte Abraham Ortelius 1590 im „Theatrum orbis terrarum“. Diese Karte ist in dem „Theatrum orbis terrarum" die einzige Karte, die eine Ortsklassifizierung mittels geometrischer Signaturen enthält. Aus diesem Grund erhielt lediglich diese Karte eine ausführliche Legende (Abb. 6). Die ursprüngliche Karte wurde bereits 1552 in Wittenberg publiziert; von ihr ist jedoch kein Exemplar überliefert. Egon Klemp entdeckte ein Fragment dieser Karte im Archiv der Stadt Plauen (vgl. Klemp, 2007), woraus ersichtlich ist, dass Stella ursprünglich die geometrischen, die Ortklassifizierung veranschaulichenden Signaturen noch mit ikonischen Symbolen kombinierte. Die Gestaltung dieser ursprünglichen Signaturen ist besonders bemerkenswert, da die zunehmende Bedeutung der Orte durch diagrammartige Addition graphischer Elemente veranschaulicht wurde. Stellas Karte wurde 1586 von Ortelius in einen kleineren Maßstab umgesetzt und 1590 publiziert. Dabei wurde auf 


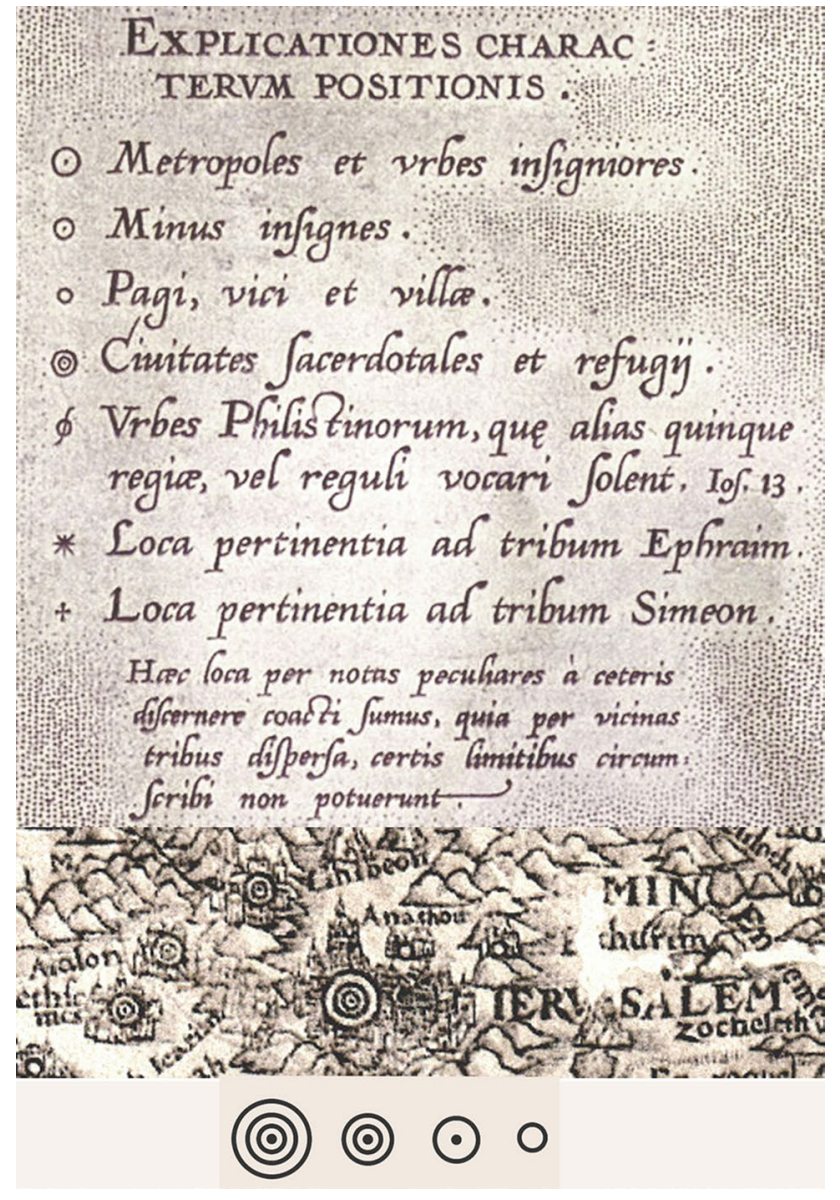

Abb. 6 Oben: Legende zur Karte von Judäa und Israel von Tilemann Stella im „Theatrum orbis terrarum“(1590); Mitte: Ausschnitt aus dem Kartenfragment (1552); Unten: Signaturen in dem Kartenfragment (von Verf. nachgezeichnet)

die ikonische Kombination verzichtet. Das Kartenfragment und die Karte im „Theatrum orbis terrarum“ stellen somit äußerst wichtige Quellen zur Genese geometrischer Kartenzeichen dar, deren Bedeutung bisher unbeachtet blieb.

\section{Schlussbemerkung}

In diesem Beitrag wurden nur jene Aspekte der vielfältigen Tätigkeit Stellas erwähnt, zu denen neue Erkenntnisse vorliegen. Die Archive in Schwerin, Dresden und Aberdeen enthalten mehrere Quellen zum Wirken Stellas, die noch nicht hinreichend ausgewertet wurden. Die Nutzung dieser Quellen wird z. T. auch durch objektive Schwierigkeiten verhindert. Die Karten Stellas, die in dem Landeshauptarchiv Schwerin aufbewahrt werden, gehören zu den Meisterwerken der Kartographiegeschichte. Sie sind für die Forschung nicht zugänglich, da bisher keine finanziellen Mittel zu ihrer Restaurierung eingeworben werden konnten.

Open Access This article is distributed under the terms of the Creative Commons Attribution 4.0 International License (http://creativeco mmons.org/licenses/by/4.0/), which permits unrestricted use, distribution, and reproduction in any medium, provided you give appropriate credit to the original author(s) and the source, provide a link to the Creative Commons license, and indicate if changes were made.

\section{Literatur}

Allgemeines Lexikon der bildenden Künstler von der Antike bis zur Gegenwart, begründet von U. Thieme und F. Becker 1907, Bd. 37: Meister mit Notnamen und Monogrammisten (1999), Leipzig

Brichzin H (1990) Tilemann Stellas Beziehungen zu Sachsen und zu Kurfürst August. In: Tilemann Stella und die wissenschaftliche Erforschung Mecklenburgs in der Geschichte (Rostocker Wissenschaftshist. Manuskripte 18). S. 29-30

Brichzin H (1993) Der Kartograph Tilemann Stella (1525-1589). Seine Beziehungen zu Sachsen und zu Kurfürst August anhand neuer Quellenfunde. In: Archivmitteilungen. Zeitschrift für Archivwesen, archivalische Quellenkunde und historische Hilfswissenschaften, 42. Jg., Heft 6, S. 211-245

Cordshagen Chr (1986) Neue Erkenntnisse zum Wirken Tilemann Stellas als Kartograph in Mecklenburg. In: Archivmitteilungen 36 Heft 5, S. 158-161

Cordshagen Chr (1989) Tilemann Stellas Wirken in Mecklenburg. Theoretische Schriften und deren Umsetzung in seinen Karten. In: Siegerland 66, 3-4, S. 83-87

Cordshagen Chr (1990) Tilemann Stellas Wirken in Mecklenburgseine theoretischen Schriften und deren Umsetzung. In: Tilemann Stella und die wissenschaftliche Erforschung Mecklenburgs in der Geschichte (Rostocker Wissenschaftshist. Manuskripte 18). S. $13-17$

Fauser A (1973) Ein Tilemann Stella-Himmelsglobus in Weißenburg in Bayern. In: Der Globusfreund, Wiss. Zs. für Globen- und Instrumentenkunde, Nr. 21/23, S. 150-155

Grenacher F (1968) Der sog. St.-Galler Globus im Schweiz. Landesmuseum. Vermutungen über seine Herkunft und Feststellungen zu seiner Konstruktion. In: Zeitschrift für schweizerische Archäologie und Kunstgeschichte. Band 21, 1961, S. 66-78

Hamel J (2018) Der Himmelsglobus Tilemann Stellas im Weißenburger Reichsstadtmuseum. Weißenburger Blätter, villa nostra 2(2018):30-35

Kaiserliches Druckprivileg für Tilemann Stella 1560. Österreichisches Staatsarchiv, Wien: Haus-, Hof- und Staatsarchiv, Bestand Reichshofrat, Impressorien Bd. 69. fol. 4. (vgl. Meurer 2001, S. 330.)

Klemp E (2007) Tilemann Stellas Karte vom Heiligen Land. Mappæ antiquæ. Utrechtse historisch-cartographische studies 6:339-344

Meurer PH (2001) Corpus der älteren Germania-Karten: Ein annotierter Katalog der gedruckten Gesamtkarten des deutschen Raumes von den Anfängen bis um 1650. Uitg. Canaletto, Alphen aan den Rijn

Oehme, R./Zögner L (1989) Tilemann Stella (1525-1589). Der Kartograph der Ämter Zweibrücken und Kirkel des Herzogtums Pfalz-Zweibrücken: Leben und Werk zwischen Wittenberg, 
Mecklenburg und Zweibrücken. Lüneburg [u.a.]: Verl. Nordostdt. Kulturwerk

Pápay G (1989) Ein berühmter Kartograph des 16. Jahrhunderts in Mecklenburg: Leben und Werk Tilemann Stellas (1525-1589). In: Beiträge zur Kulturgeschichte Mecklenburgs aus Wissenschaft und Technik (= Rostocker Wissenschaftshistorische Manuskripte 12). S. $17-24$

Pápay G (1990) Tilemann Stella und die Kartographie des 16. Jahrhunderts. In: Tilemann Stella und die wissenschaftliche Erforschung Mecklenburgs in der Geschichte (Rostocker Wissenschaftshist. Manuskripte 18), S. 7-12

Pápay G (2016) Gutachten zu dem St. Gallener Globus (Manuskript vom 06.09.2016)

Pápay G (2018) Der Weißenburger Himmelsglobus im Kontext der Globuskartographie von Tilemann Stella. Weißenburger Blätter, villa nostra 2(2018):5-29

Rohrbach, M./Gnädinger, B. (Hrsg.) (2009): Der Zürcher Globus. Projekt Globus-Replik 2007-2009, Dokumentation, Zürich: Staatsarchiv des Kantons Zürich, 2009. https://staatsarchiv.zh.ch/inter net/justiz_inneres/sta/de/ueber_uns/veroeffentlichungen/_jcr_ content/contentPar/downloadlist/downloaditems/download.spool er.download.1282816347217.pdf/Globus_Doku_1_0.pdf. Letzter Zugriff: 20. Oktober 2018

Schmid J (2016) A previous unknown likeness of the St Gallen Globe: new speculations about its origin. J Int Map Collect Soc 144:12-21
Schmid J (2017) Optische und radiografische Analysen zum St. Galler Globus (1576). Neue Erkenntnisse zur seiner Datierung und Urheberschaft.- In: Zeitschrift für schweizerische Archäologie und Kunstgeschichte. Heft 2, S. 145-156

Schmid-Lanter J (2018) Der kosmographische St. Galler Globus. Cartographica Helvetica, Heft 56:35-44

Stella Tilemann Sigenensis (1566) Methodvs, qvæ in chorographica et historica totius germaniæ descriptione obseruabitur. Rostock: Jakob Lucius. (Das Verfahren von Tilemann Stella aus Siegen, das bei der chorographischen und historischen Beschreibung von ganz Deutschland beachtet werden wird.) Die Übersetzung wurde von P. H. Meurer 2001 veröffentlicht (vgl. Meurer 2001, Anhang 5-2)

Stella, Tilemann von Sigen (1560) Kurtzer und klarer bericht vom gebrauch und nutz der newen Landtaffeln sampt iren zugeordenten scheiben oder circkeln in etliche capittel verfasset dem günstigen leser zu gut. Wittenberg, Johann Krafft, 1560, 1562, 1563; Peter Seitz 1567, 1571; Clement Schleich 1580; Gregor Braun 1590, 1595

Stewart AM (1973/74) Tilemann Stella of Siegen. Aberdeen University MS 149. In: Aberdeen University Review, vol. 45, S. 179-183

Vicentius, Petrus (1554) Clarissimo viro d. Tilemanno Stallae mathematco et geographo excellenti epithamion scriptum, Rostock

Voltelini H (1894) Urkunden und Regesten aus dem k.u.k. Hausund Staatsarchiv in Wien. In: Jahrbuch der kunsthistorischen Sammlungen des Allerhöchsten Kaiserhauses 15, Wien. (Nachdruck Graz 1966) 